\title{
A Simulation Model of Big Data Analysis for Fire Alarm
}

\author{
Shi-jiao ZHU ${ }^{1, a}$,Jia-Yao Zhang ${ }^{1, b}$,Xing Zhou ${ }^{2, c}$, JunYang $^{2, c}$ \\ ${ }^{1}$ School of Computer and Information Engineering, Shanghai University of \\ Electric Power, Shanghai 200090, China \\ ${ }^{2}$ Dimu Technology Ltd,Shanghai,China \\ azhusj707@hotmail.com, ${ }^{\text {b } 754351426 @ q q . c o m, ~}{ }^{\mathrm{c}} 754351426 @ 163 . c o m$
}

Keywords: Big data.Video Analysis,Alarm system.

Abstract. This paper discusses the construction and implementation of forest early warning system from the perspective of large data storage, analysis and structure. In this paper, the architecture and model of the combination of video alert and large data forecast are proposed, and it analyzes and discusses it. Experimental results show that the method has good expansibility and superiority.

\section{Introduction}

The rapid development of the network, provides a wide range for the application of big data, which is the most popular research field [1]. Big data has been widely used in the field of transportation and other applications, but not much for the prevention and early warning [2]. From the big data model, this paper tries to apply the complex early warning alarm system combing with big data platform[3]. Forest fire warning is the main application field of the current security [4] [5]. Video fire alert system is a fast and effective way for such application which is also active for the study of early warning [6].

All of these requirements need to handle large amounts of data from sensing devices, such as video devices. Big data model has the characteristics of security and stability which can suitable for more complex application scenarios and can guarantee the stability of the system for a long time. In addition, because of the time period of video analysis is longer, the calculation which based on big data can meet the requirement well, and the video based analysis can achieve the high cost performance of the sensing device. For its convenient and shortcut, people does not need to make any physical contact with it.

The paper is organized as follow. Definitions and operators necessary for description of the customized housing image are provides in Section 2. This discussion is followed by the description of the proposed algorithm in Section 3. Results of images and comparison with different smokes are providing in Section 4, with conclusions in Section 5.

\section{Model}

The discovery of the image data it originates from the judgment of the abnormal data. In this paper, we do a research on the basic model and judge, and give a concrete video forecast model.The basic judging unit can be implanted into the big data model, which is convenient for the regularity of the discovery of data during the late application. The paper defines the basic symbols as follows:

- $\quad R^{n}$ :Denote the n-dimensional Euclidean space characteristics;

- $S_{i}$ : Said the first I feature subspace in n-dimensional space, where $S_{i} \subset R^{n}, i \in\{1,2 . . m\}$;

- $\left\{\psi_{i}^{k}\right\}$ : Collection of K class ,I judgment, $k \in I, i \in\{1 \ldots m\}$; individual judgment unit $\psi_{i, j}^{k}$, means the judgment of K class, J layer, I unit.

- $L_{k}$ : Determine the collection by the k layer, define $L_{k}:=\left\{\left\{\psi_{i}^{k}\right\} \mid k \in I, i \in\{1 \ldots m\}\right\}$;

- $O_{k}$ :Determine the output sequence of the $\mathrm{k}$ layer, define $O_{k}:=\left\{0, \ldots 1_{q}^{p}, 0,0 \mid q \in\{1 . m\}, p=0 o r 1\right\}$ 
- $\quad Y_{i}$ :Discriminant the output of class I.

The unit model of this judgment accords with the basic data of large data calculation, which can be used by different nodes, and statute the large data into small data.

The concept is embodied in the network layer structure as shown in Figure 1.

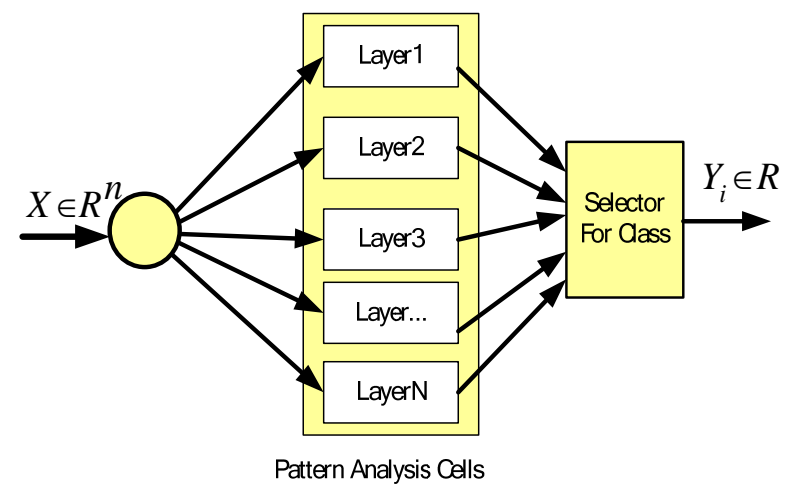

Figure 1 Data Analyis Units

The judging unit finally gives the early warning and judgment of the vote through the internal data node and the input of different data, which combined with the existing data analysis model. This process has the characteristics of independent parallel operation.

Basic Principle of the Structure of Early Warning Model

For video picture analysis unit which defined as $\psi_{i, j}^{k}$, stipulate that the individual analysis unit $\psi_{i, j}^{k}$ a node in the large data model, which's stage includes many attribute features. To express these features by means of numbers, the measurement of the similarity is described as follows.

$$
F(\rho)=\left\{\begin{array}{l}
1, \rho<\Gamma_{0} \\
0, \text { else }
\end{array}\right.
$$

As judging by sample structure, $\psi_{i, j}^{k}$ has the judgment element with the same level is defined as $C\left(\psi_{i}^{k}\right)$, in which the coverage area of different nodes is not overlapped. $C\left(\psi_{i}^{k 1}\right) \cap C\left(\psi_{i}^{k 2}\right)=\phi, k 1 \neq k 2$ Representation of nodes and different computational elements in the same situation.

When constructing the big data internal judgment model, thinking fully about the effective attribute range of early warning judgment. In the initial stage of the operation the range of node coverage is limited, but with the continuous data entering, the overlay unit and the overlay layer are increasing, and the collection of the covering nodes is formed. The function range of the effective features in the large data model is reflected by the collection.

The network acceptance data $x \in R^{n}$ which waits for input. Sequence of neuron output response of each neuron $O_{k}$. Definition discrimination:

$$
\begin{aligned}
& Y_{i}:=\left\{i \mid i=\left\{0 \text { org }:=\left\{\operatorname { m i n } ( k ) \& \left\{O_{k}>1\right.\right. \text { The feature partition of the early warning is }\right.\right. \\
& \rightarrow p\}\}\}\}
\end{aligned}
$$

divided by $Y_{i}$ to the sample $x \in R^{n}$ which waits for identify. $Y_{i}=\left\{\begin{array}{l}\text { category } i, i>0 \\ \text { refuse, } i=0\end{array}\right.$

From the type of category recognition network, warning judgment can effectively avoid "false negatives", and this is suitable for the characteristics of early warning 


\section{System Framework}

The system structure is based on the early warning and the data analysis and forecast are characterized.System, give full consideration to the practical application in forest fire monitoring, and supplemented by the protection of natural ecology. System also applying the new technology to the forest resources management system such as the GIS technology, thenanoscale electronic waves filter technology, intelligent video analysis, 3G/WI-FI wireless transmission, digital image processing technique and the integrated application of high technology. The early warning framework is based on the big data. It has characteristics of fast responseand can realize the forest fire monitoring which is based on theanalysis of image data. The topological diagram of the system is as follows:

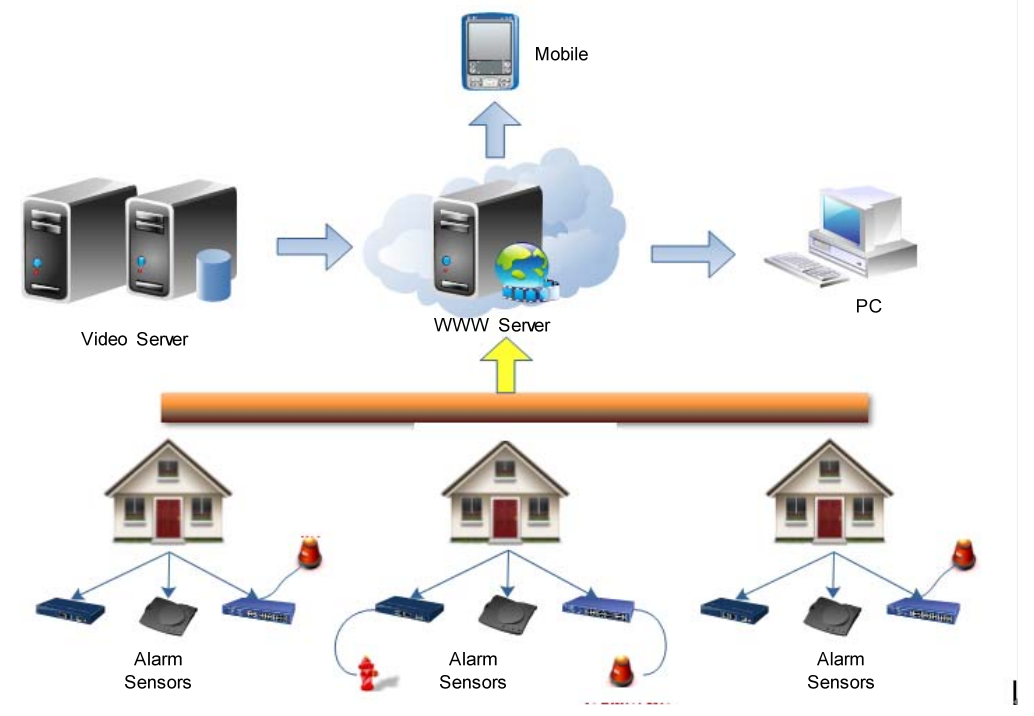

Figure 2 system architecture diagram

The model defines each terminal station equipment which has independent data address coding, in order to facilitate the system infrastructure; and with GPS positioning based on independent code, it can achieve a unified GPS equipment display system, which is convenient and quick. At the same time,the front equipmentget the video data acquisition bythe non-contact video equipment, which has the intelligence characteristic for the forecast of video data, and the framework anticipation the data technology, when the disaster warning occurs, the system achieve the best rescue method through the calculation of the fast data, which is faster than before. However, the basic of these operations is the parallel computing technology of large data platform and video analysis. To improve the reliability of the system. When emergency occurs, the system will send messages to alert the person in charge of the project in order to convenient the artificial judgment to add the reliability of the system and it is convenient for leaders and experts to guidance and scheduling in time.

The model uses the monitoring method of digital equipment. It transferthe information and datato the forestry monitoring and command centerthrough wireless network using the GIS (Geographic Information System) to achieve the positioning of fire, andmake the analysis judgment timely to determine the suppression project,and control the fire in the bud. At the same time, it can storage, process and analyse a large amount of data. This will guide the future forest fire prevention work and reference the decision-making value.

Access to information:Using the field information acquisition station, which is established and is distributed onthe different high points in the fire prone areastogain surveillance video images within the coverage, to realize the all-weather uninterrupted monitoring.

Dynamic monitoring: It can transfer the video image and other information to the District Fire Monitoring Center timely and synchronously under the support of the wireless digital network platform system, to realize the dynamic situation of the true observation forest region.

Fire warning:In case of fire, it usethe geographic information system (GIS) to get relevant data to understand and master the basic situation of the field of fire, in order to realize accurate positioning. At the same time, it concludes a set of feasible fire suppression scheme and the number of people, 
machine and material strength of configuration, and then come out the suppression of specific measures and the best route scheme, through the analysis of forestry professional database.

Forecast analysis:Predict the forest fire grade data of the region by referencethe forest phenology and combustible property data and by analysis using expert database model comprehensively.

The fire prediction system which combine the videoavoid the original artificial fire lookout observation limitations, that realizes the digitization and scientific of the forest management. It greatly reduce the cost of the forestry sector and management costs and improve the effect of the forest enterprise. When monitoring the forest fires, the system can also monitor the forest resources, ecological environment, plant diseases and insect pests of forest and wildlife and deforestation and other forestry activities effectively.

The system is assumed to be based on the Linux system and use Hadoop as the system platform. The hierarchical memory system architecture design, lay the foundation for the follow-up data mining and data acquisition module design and implementation anduses the HBase for data storage. The system structure as shown:

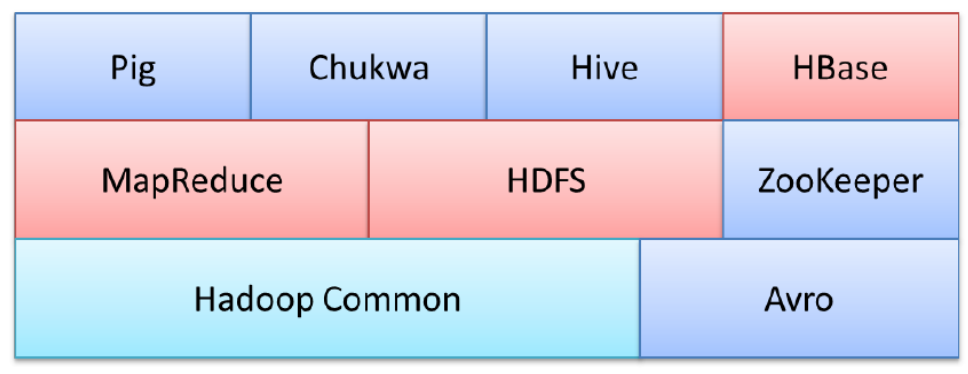

Figure 3 Architecture of Big Data

The system architecture: In the system architecture isbased on the application of large data, which adapt the Hadoop platform (defined as JHadoop) and MapReduce technology. It use the parallel computer system, fault-tolerant processing mechanism and job scheduling mechanism. It also use some key technologies of HBase, such as CAP principle, query model, data version, data query, MapReduce based data processing, HBase and relational database, etc..Finally, it introduces and analyzesthe key technology of HDFS and introduces the architecture and data of HDFS as a key.

The storage:The system must be integrated with the architecture of the hierarchical storage system in the storage to lay the foundation for the design and implementation of the following data mining and data acquisition module.

In the aspect of data acquisition, the data acquisition module of the hierarchical storage system is designed and implemented.Aiming at the different features of the structured and unstructured data, a targeted solution is proposed in this paper

For structured data, this scheme shows that the data from the traditional relational database based on the MapReduce framework of the collection scheme to collect the HBase and to optimize the design for the MapReduce scheduler to improve the collection efficiency. Because data acquisition is IO intensive operation, the scheduling scheme is based on IO occupancy rate in architecture. According to the scheduling, the algorithm of the scheme is used to carry out the task scheduling to ensure that the new task can be allocated to the lower IO load nodes, to improve the task execution efficiency.The introduction of program supports manual input.

\section{Experiment}

The actual experiment was carried out in a forest accident center in Jiangxi province and judged the related natural fire. The experiment uses the classification analysis of the natural fire video pictures and combines the basic definition of large data to deal with the data quickly.

In order to observe the impact of the model in different environments the video frequency is about average about 200 seconds, and the average video is about 400 seconds. The following tests were averaged and the correct rate is defined as the proportion of the time point to the end of the fire. 
Table 1 the Effect of Fire Alarm Set to the Early Warning Video

\begin{tabular}{|c|c|c|c|c|}
\hline Fire Type & $\begin{array}{c}\text { Correct } \\
\text { rate (\%) }\end{array}$ & Error rate (\%) & Rejection rate (\%) & Time (seconds) \\
\hline Fire Video Outdoor & 90.75 & 9.25 & 0 & 210 \\
\hline Fire Video Indoor & 92.84 & 7.16 & 0 & 400 \\
\hline
\end{tabular}

Through the analysis of table 1 it can be seen that the image analysis of this system is considerable stable.Whether in simulation environment or experimental environment both the rejection rate was 0 . It can alarm the fire warning effectively Which can guarantee the personal safety under the reality and protect the personal safety in real situations.

\section{Conclusions}

This paper studies the fire alarm system of video in large data environment. It discussed the fusion of the video image and the big data timely, and the parallel timeliness of the analysis of video of the description of the Mapreduce nodes in the big data. The example shows that:

- Big data in practical application as well as in the application of image analysis environment is especially for high stability fire alarm system;

- The big data platform can provide a good platform for the image and subsequent analysis.

The development of large data platform technology is more consistent with the current fire prevention network needs, and can overcome the single node instability features. It can give play to the advantages of large data applications fully. In the fact of using big data toanalyse and predict data is worth the next step.

\section{Acknowledgement}

The Project was supported by the Natural Science Foundation of Shanghai,China(Grant No. 13ZR1417200). The authors are grateful for the anonymous reviewers who made constructive comments.

\section{References}

[1] Min Chen,Shiwen Mao,Yunhao Liu. Big Data: A Survey,Mobile Networks and Applications, April 2014, Volume 19, Issue 2, pp 171-209.

[2] Lomotey RK, Deters R (2014) Towards knowledge discovery in big data. In: Proceeding of the 8th international symposium on service oriented system engineering. IEEE Computer Society, pp 181-191

[3] CRAGLIA M, BIE K, JACKSON D, Digital Earth 2020:Towards the Vision for the Next Decade [J] . International Journal of Digital Earth,2012,5(1):4-21.

[4] Rossi, L., Akhloufi, M., Tison, Y.: On the use of stereovision to develop a novel instrumentation system to extract geometric fire fronts characteristics. Fire Saf. J. (1), 9-20 (2011)

[5] Habiboglu, Y.H., Günay, O., Çetin, A.E.: Covariance matrix-based fire and flame detection method in video. Mach. Vis. Appl.23(6), 1103-1113 (2012)

[6] Elham Mahdipour - Chitra Dadkhah.Automatic fire detection based on soft computing techniques: review from 2000 to 2010 Artificial Intelligence Review,December 2014, Volume 42, Issue 4, pp 895-934. 